\title{
Direct imaging of surface plasmon polariton dispersion in gold and silver thin films
}

\author{
Megan Ives ${ }^{1}$, Travis M. Autry ${ }^{1,2}$, Steven T. Cundiff $1,2,3$, And Gä̈L Nardin ${ }^{1,4}$, $^{*}$ \\ ${ }^{1}$ JILA, University of Colorado \& National Institute of Standards and Technology, Boulder CO 80309-0440, USA \\ ${ }^{2}$ Department of Physics, University of Colorado, Boulder CO 80309-0390, USA \\ ${ }^{3}$ Department of Physics, University of Michigan, Ann Arbor, MI 48109, USA \\ ${ }^{4}$ Laboratory of Applied Photonics Devices, School of Engineering, Ecole Polytechnique Fédérale de Lausanne (EPFL), CH - 1015 Lausanne, Switzerland \\ *Corresponding author: gael.nardin@a3.epfl.ch
}

Compiled March 3, 2016

\begin{abstract}
We image the dispersion of surface plasmon polaritons in gold and silver thin films of $30 \mathrm{~nm}$ and 50 $\mathrm{nm}$ thickness, using angle-resolved white light spectroscopy in the Kretschmann geometry. Calibrated dispersion curves are obtained over a wavelength range spanning from $550 \mathrm{~nm}$ to $900 \mathrm{~nm}$. We obtain good qualitative agreement with calculated dispersion curves that take into account the thickness of the thin film. ( 2016 Optical Society of America
\end{abstract}

OCIS codes: (000) codes

http://dx.doi.org/10.1364/ao.XX.XXXXXX

\section{INTRODUCTION}

Surface Plasmon Polaritons (SPPs) are coupled modes that exist at metal-dielectric interfaces [1-3]. Their mixed character of electromagnetic waves and surface electron oscillations makes them suitable for sub-wavelength focusing, enabling nano-optical circuits, slow light, and extraordinary transmission. The Kretschmann geometry is widely used in the context of gas detection and biosensing [4-6] or to measure the complex dielectric function of the metal itself [7]. These applications rely on the sensitivity of the Surface Plasmon Resonance (SPR) to the refractive indices of the metal and the dielectric forming the interface. Using a 2D detector, SPR imaging provides the spatial distribution of refractive index of a sample using a contrast measurement at a single wavelength. SPR imaging has numerous applications in biology [8], can be implemented with phase retrieval [9], and was recently demonstrated to be used for wavefront sensing [10].

To design plasmonic devices, it is essential to know the dispersion relation of the SPP mode. SPP dispersion curves have been obtained using electron-energy loss spectroscopy measurements [11]. Using optical means, the SPR at a particular wavelength can be observed in the Kretschmann geometry, by measuring the reflected intensity of a monochromatic light source as a function of angle of incidence $[1,7,12]$. In a similar configuration, a measurement of the whole dispersion curve can be obtained by sequentially scanning the illumination wavelength [13]. Single shot mapping of SPP dispersion was achieved by acquiring the spectrally- and angle-resolved reflectivity of a white light source on a 2D charged-coupled device (CCD) array, using a prism [14] or a grating $[15,16]$ for spectral resolution.

Here we show the direct, calibrated imaging of SPP dispersions using white light reflectivity and an imaging spectrometer, over a wavelength range spanning from $550 \mathrm{~nm}$ to $900 \mathrm{~nm}$. Reflectivity maps, SPP dispersion curves and SPR widths are extracted and compared to calculations. We show that the thickness of the metallic film needs to be taken into account to achieve qualitative agreement with the experimental results.

\section{SPP MODES IN THE KRETSCHMANN CONFIGURA- TION}

The dispersion relation of a SPP at a metal-dielectric interface is [1]

$$
k_{S P P}=\frac{2 \pi}{\lambda} \sqrt{\frac{\epsilon_{m} \epsilon_{d}}{\epsilon_{m}+\epsilon_{d}}}
$$

with $\lambda$ the wavelength of light in vacuum, and $\epsilon_{m(d)}$ the complex dielectric permittivity of the metal (dielectric), generally a function of $\lambda$. The real part of this expression provides the energy of the SPP, and the imaginary part is linked to dissipation (or to the linewidth of the SPP resonance). For a metal/air interface, Eq. 1 reduces to $k_{S P P}=\frac{2 \pi}{\lambda} \sqrt{\frac{\epsilon_{m}}{\epsilon_{m}+1}}$. The SPP dispersion lies out of the light cone, and thus coupling with external light needs additional in-plane momentum. The wavevector matching condition is commonly achieved by scattering on defects, gratings, or by injecting light from a medium with higher refractive index (e.g. glass) [1], as depicted in Fig. 1 (a). In the latter case, it is required to use a metallic film with thickness $d$ smaller than the SPP penetration depth in the metal, so that the SPP at the 
metal/air interface can be coupled to light incoming from the glass side. In this paper we couple light to SPPs confined in thin films deposited on the hypothenuse of $B K 7$ prisms, in the Kretschmann configuration.

\section{EXPERIMENTAL SETUP}

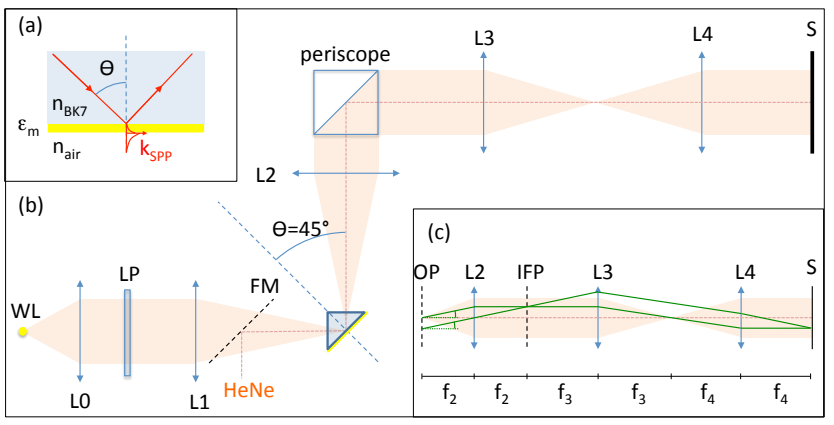

Fig. 1. (a) Coupling of SPPs with external light, incident on the thin film with an angle $\theta$ in a medium with refractive index larger than unity. The SPP is created a the air/metal interface, carrying a wavevector $k_{S P P}$. (b) Experimental setup for angle-resolved white light reflection spectroscopy in the Kretschmann geometry (described in text). WL: white light source, L0-L4: lenses, LP: linear polarizer, FM: mirror on a flip mount, S: entrance slit of the spectrometer. (c) Detection part of the experiment, unwrapped. Light coming from the object plane (OP) forms an intermediary Fourier plane (IFP), which is then imaged on the spectrometer slit $(\mathrm{S})$. Therefore, light rays coming from the OP with same angle are focused on a same point in the $S$ plane. $f_{2}-f_{4}$ : focal lengths of lenses $L 2-L 4$

The experimental setup is illustrated in Fig. 1 (b). We used $B K 7$ right angle prisms (10mm long right angle facets - Thorlabs PS910), on which we deposited thin films of gold and silver, using e-beam evaporation ( $5 \%$ uncertainty on layer thickness). Four prisms were coated, two with each metal, with two different thicknesses: $30 \mathrm{~nm}$ and $50 \mathrm{~nm}$. The prisms were mounted on a rotation stage (precision: 5 arcmin). The reflectivity experiment was performed with a white light source (150 illuminator, RAM optical instruments) collimated into a $25 \mathrm{~mm}$ diameter beam. A linear polarizer was used to control the polarization of the white light. Lens $L 1$ (focal length $f_{1}=8 \mathrm{~cm}$ ) focuses light on the thin film through the right angle facet. The reflected light was collected by lens $L 2$ (achromatic doublet, focal length $f_{2}=8 \mathrm{~cm}$ ), and directed towards an imaging spectrometer via a periscope and lenses $L 3$ and $L 4\left(f_{3}=20 \mathrm{~cm}, f_{4}=10 \mathrm{~cm}\right)$. The role of lenses $L 2-L 4$, as depicted in the unwrapped sketch of Fig. 1 (c), is to create an intermediary Fourier plane, which is then imaged on the entrance slit of the spectrometer (slit width: $50 \mu \mathrm{m}$ ). In this way, the angle at which the light is reflected from the thin film is mapped on the plane of the spectrometer entrance slit. The role of the periscope, whose two mirror main axes are rotated by $90^{\circ}$ with respect to each other, is to rotate the light propagation plane by $90^{\circ}$. Therefore, the horizontal angular spread of light reflected from the thin film is vertically spread along the spectrometer slit. At the output of the spectrometer, the CCD records $2 \mathrm{D}$ images (the two axes being wavelength and reflected angle), thus directly mapping the SPP dispersion. To increase the throughput (e.g. in case of a lower-power light source or to achieve shorter acquisition times), it would be possible to use a cylindrical lens in place of L3, such that the lens L4 would focus light into a stripe that could be aligned on the slit of the spectrometer.

Our implementation of direct SPP dispersion mapping has the following advantages over previous realizations [14-16]: (1) The imaging of the Fourier plane on the spectrometer slit ensures that angular resolution is not lowered due to the size of the illuminated area on the thin film (which in turn is linked to the size of the white light source). In our case, with an illumination spot of $\sim 1 \mathrm{~mm}$ diameter and $f_{2}=8 \mathrm{~cm}$, we obtain an angle resolution of $0.1^{\circ}$. (2) The spectral resolution is set by the spectrometer and its entrance slit and is not lowered by off-axis propagation as in the case of a beam initially filtered by a slit prior to illuminating the thin film.

\section{CALIBRATION}

In this section we describe the calibration of wavelength, angle, and reflectance. This calibration provides information on the position, width and depth of the SPP resonance, and enables extraction of calibrated dispersion curves.

\section{A. Calibration of wavelength}

The wavelength is calibrated using a Krypton lamp. The spectrometer (1/4 m Digikröm, CVI) uses a grating with 600 lines per $\mathrm{mm}$, providing a wavelength spread of $\sim 60 \mathrm{~nm}$ across the CCD. Rotating the grating, a series of seven acquisitions was taken to cover the wavelength range $550 \mathrm{~nm}-900 \mathrm{~nm}$.

\section{B. Calibration of angle}

A collimated HeNe laser beam was used to calibrate the angle axis. To avoid having refraction in the prism perturb the calibration process, we rotated it by $180^{\circ}$ so that the HeNe laser was incident on the metal/air interface. From this side, there is no plasmonic effect and the laser beam is simply reflected on the metal surface. Rotating the prism using the precision rotation stage, the laser beam focused at different heights on the CCD, providing, in first approximation, a linear relation between angles and CCD pixels. To obtain the CCD pixel row for which the light is reflected from the thin film at an angle of $\theta=45^{\circ}$, we position the prism such that the HeNe laser is back-reflected from the prism facet. We estimate the uncertainty on this angle offset to be less than $0.25^{\circ}$. We then slightly rotate the prism to cover the angle range $39.3^{\circ}-48.7^{\circ}$. Note that angles measured with this calibration correspond to angles in air. Therefore, results showed in this paper are plotted as a function of $\theta_{\text {air }}$. Since refraction occurs at the facet of the prism (which is at $45^{\circ}$ from the film), a small correction given by Snell's law needs to be applied to find the angle $\theta$ at which the light is reflected from the thin film in $B K 7$

$$
\theta=\sin ^{-1}\left(\frac{1}{n_{B K 7}} \sin \left(\theta_{\text {air }}-45^{\circ}\right)\right)+45^{\circ}
$$

where $n_{B K 7}$ is the refractive index of $B K 7$.

In our configuration, the numerical aperture of the experiment is limited by the diameter of $L 2(25 \mathrm{~mm})$ to a range of $\sim 17$ degrees $^{1}$. However, we choose focal lengths $f_{2}-f_{4}$ to obtain a magnification of the intermediate Fourier plane such that an angular spread of $\sim 9.5^{\circ}$ is obtained across the CCD. This spread is large enough to track the SPP dispersion over the studied wavelength range.

\footnotetext{
${ }^{1}$ To cover a much wider angle range, larger NA optics and a hemi-cylindrical prism should be used, as demonstrated by Park et al. [16]
} 


\section{Normalization of reflectivity}

We acquired for each wavelength range a reflection map with TEand TM-polarized light. Since SPPs do not exist for TE polarization [3], we used the TE map to normalize the TM measurement. Fresnel reflections on the prism facets are almost identical for $T E$ and TM polarization, since the angle of incidence on the facets is less than $5^{\circ}$ from normal. Therefore, the TM/TE normalization allows us to highlight the SPR, while normalizing the white light spectrum and eliminating measurement artifacts such as dust on the optics. An example is given in Fig. 2. Since the TE reflectance is smooth and within $15 \%$ of unity on the range of interest, the normalization does not impact the measurement of the SPR angle and width.

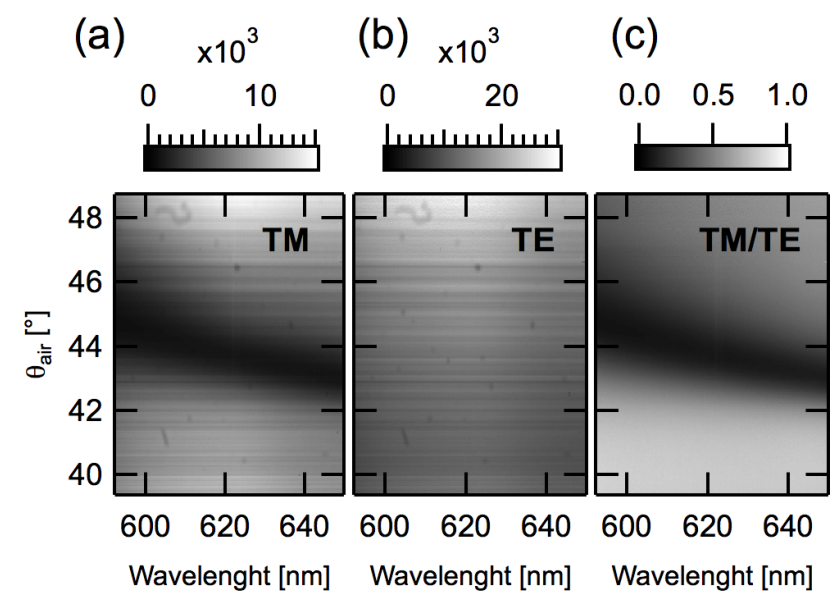

Fig. 2. (a) Raw reflectivity data measured for $T M$-polarized light. (b) Same for TE-polarized light. (c) Ratio of TM and $T E$ measurements. Grayscales are given as a number of CCD counts for (a) and (b). In (c), the TM/TE ratio is unitless, with grayscale from 0 (black) to 1 (white).

\section{RESULTS}

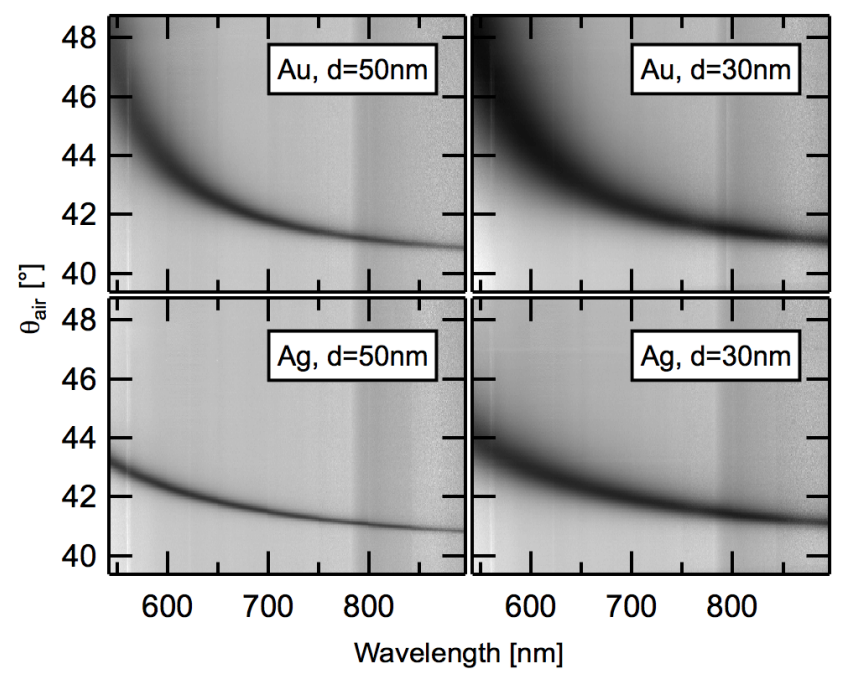

Fig. 3. Measured reflectivity maps (TM/TE) as a function of reflection angle $\theta_{\text {air }}$ and wavelength, for four different thin films. The linear grayscale goes from 0 (black) to 1 (white).

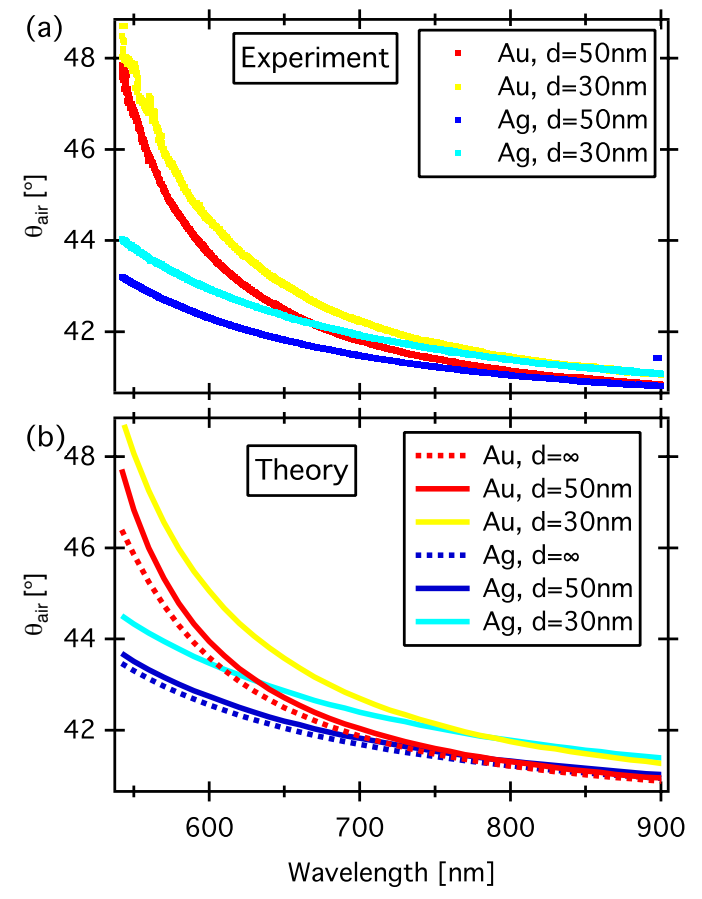

Fig. 4. (a) Experimental SPP dispersion curves, extracted from the reflectivity maps of Fig. 3. (b) Calculated SPP dispersion curves. Dotted lines $(d=\infty)$ are obtained from Eq. 1 (single interface). Solid lines are extracted from calculated reflectance maps of Fig 5, taking into account the two interfaces of the thin film.

The reflectivity maps (TM/TE) as a function of wavelength and reflection angle, measured and calibrated as described above, are shown in Fig. 3, for four prisms coated with thin films of gold and silver with thicknesses $d=50 \mathrm{~nm}$ and $d=30$ $\mathrm{nm}$. The SPP mode is clearly visible in all four thin films. A qualitative observation is that the linewidth is larger (both on the angle and the wavelength axes) for the gold SPP compared to silver, and for the thinner films. A larger linewidth corresponds to a larger SPP damping rate (and therefore shorter propagation length). The larger losses for the thinner films can be understood in terms of increased leakage radiation. The larger damping for gold can be linked to a closer proximity of the plasma frequency in this material [3]. SPP dispersion curves are extracted as the minima of the reflectivity maps, and displayed in Fig. 4 (a). The angle $\theta$ at which the the light was reflected from the thin film in $B K 7$ can be obtained from $\theta_{\text {air }}$ using Eq. 2. To obtain the dispersion in an energy $(E)$ vs in-plane momentum $\left(k_{/ /}\right)$plot, the relations $E=\frac{h c}{\lambda}$ (with $h$ the Planck constant and $c$ the speed of light in vacuum), and $k_{/ /}=\frac{2 \pi}{\lambda} n_{B K 7}(\lambda) \sin (\theta)$ can be used.

\section{COMPARISON WITH THEORY}

In Fig. 4 (b) we plot dispersion curves obtained from theory. The (complex) indices of refraction are obtained from Refs [17] (gold), [18] (silver), and [19] (BK7). First, we plot the dispersion curves obtained from Eq. 1 (dotted lines). This equation only describes the SPP mode of a single metal/dielectric interface. Therefore it corresponds to a semi-infinite metallic film $(d=\infty)$. To account for the finite thickness of the film we use Fresnel equations for an assembly of two parallel interfaces, as described in Refs [20, 21]. 


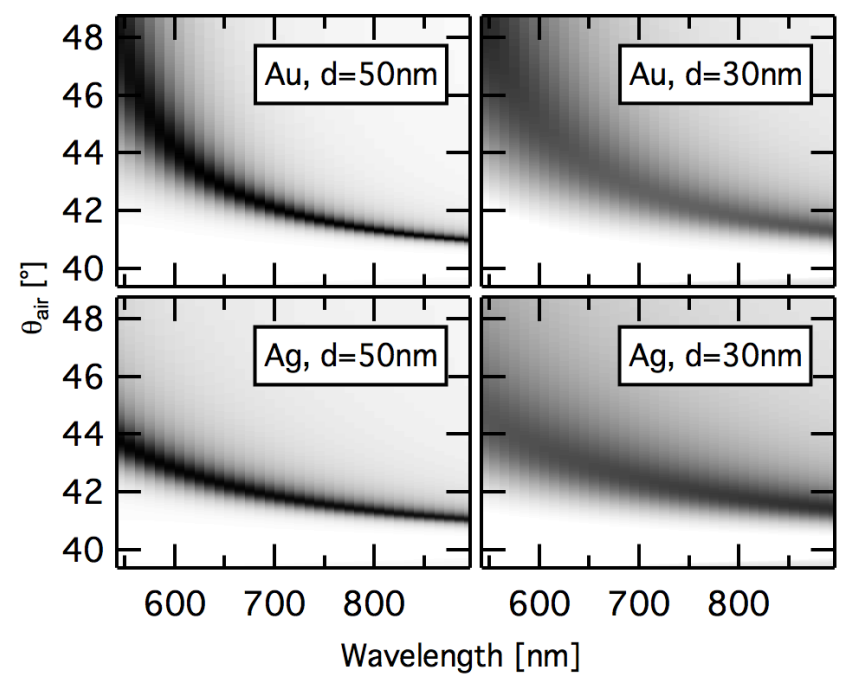

Fig. 5. Calculated maps of the TM/TE reflectivity ratio, as a function of reflection angle $\theta_{\text {air }}$ and wavelength, for four different thin films. Calculated according to Eq. 3. The linear grayscale goes from 0 (black) to 1 (white).

The reflectance map $R(\theta, \lambda)$ is

$$
R=\left|\frac{r_{12}+r_{23} e^{2 i k d}}{1+r_{12} r_{23} e^{2 i k d}}\right|^{2}
$$

with

$$
k=\frac{2 \pi}{\lambda}\left(\epsilon_{m}(\lambda)-n_{B K 7}^{2}(\lambda) \sin ^{2}(\theta)\right)^{\frac{1}{2}}
$$

and $r_{12}, r_{23}$ are the Fresnel reflection coefficients at the $B K 7 /$ metal interface and metal/air interface, respectively. They are

$$
\begin{aligned}
& r_{i j}=\frac{n_{i} \cos \left(\theta_{i}\right)-n_{j} \cos \left(\theta_{j}\right)}{n_{i} \cos \left(\theta_{i}\right)+n_{j} \cos \left(\theta_{j}\right)} \text { for } T E \\
& r_{i j}=\frac{n_{j} \cos \left(\theta_{i}\right)-n_{i} \cos \left(\theta_{j}\right)}{n_{j} \cos \left(\theta_{i}\right)+n_{i} \cos \left(\theta_{j}\right)} \text { for TM }
\end{aligned}
$$

where at each interface $\theta_{i(j)}$ is the angle of the incoming (refracted) light, obtained from $\theta_{1}$ using Snell's law, and $n_{2}=\sqrt{\epsilon_{m}}$ is the complex refractive index of the metal. The ratio of reflectance maps for gold and silver layers of $30 \mathrm{~nm}$ and $50 \mathrm{~nm}$ thicknesses, $\frac{R_{T M}}{R_{T E}}$, are displayed as a function of $\theta_{\text {air }}$ and $\lambda$ in Fig. 5 for comparison with experimental results. Overall, a good qualitative agreement between theory and experiment is observed. The minima of the SPP reflectance are tracked and displayed in Fig. 4 (b). We see excellent qualitative agreement with measured dispersion curves: the SPP resonance angle increases for decreasing thickness $d$. Comparing the obtained dispersion curves with those described by Eq. 1, we see that it is essential to take into account the finite thickness of the metallic film to obtain agreement with experimental results. A slight quantitative mismatch (up to half a degree) between experiment and theory can be observed for some parts of the curves, and may be due to chromatic aberrations in the measurement (in addition to the $0.25^{\circ}$ uncertainty on the prism positioning mentioned earlier).

Then we also measure the width of the SPR as a function of wavelength, for experimental and simulated data. The result is shown in Fig. 6. A qualitative agreement between theory and experiment is obtained: the trends as a function of wavelength are similar ; the SPR width is wider for gold than silver, and for thinner films. Quantitatively, the measured widths are generally narrower than the simulated ones. The quantitative discrepancy may be due to cumulated uncertainties on the film thicknesses, the optical alignment, chromatic aberrations, and the imaginary part of the metal dielectric constants (significant discrepancies can be found in the literature [17]).

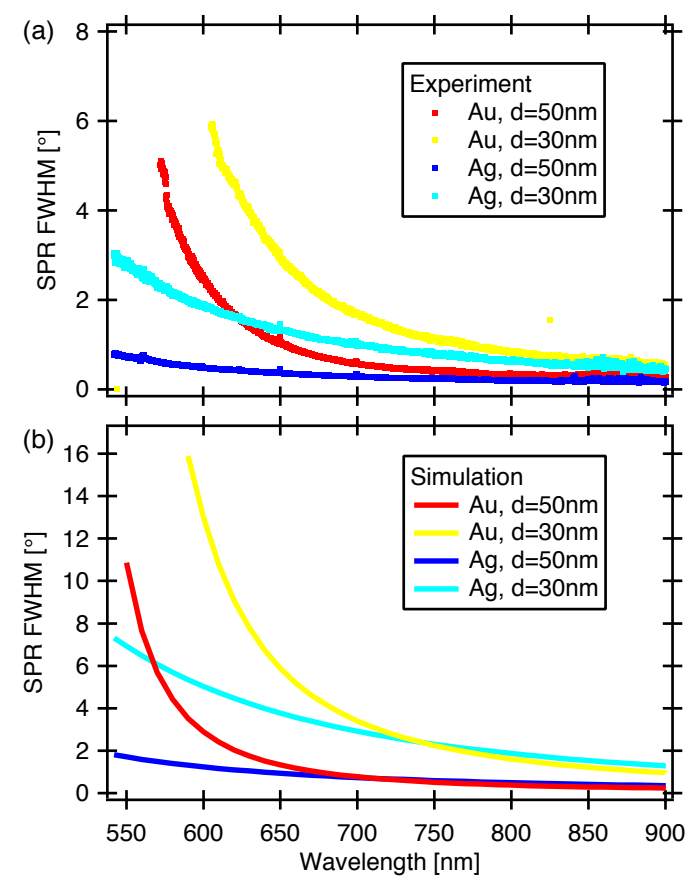

Fig. 6. Full Width at Half-Maximum (FWHM) of the Surface Plasmon resonance (SPR) extracted from (a) experimental data, and (b) simulated data.

\section{CONCLUSION}

In conclusion, we have used angle-resolved white light reflection spectroscopy in the Kretschmann configuration to directly obtain maps of the SPP modes as a function of angle and wavelength. From the reflectivity maps we have extracted calibrated SPP dispersion curves for gold and silver thin films. The careful calibration and high resolution of the experimental results allow us to detect the effect of the film thickness on the dispersion curve. We obtain qualitative agreement with theoretical calculations that take into account the finite thickness of the thin film. We have provided detailed explanations on the measurement method, calibration procedures and calculations, so that the experiment can be easily reproduced to measure calibrated SPP dispersion curves in smooth, rough or nano-structured thin films made of various plasmonic materials.

\section{ACKNOWLEDGEMENT}

We thank D. Alchenberger for the deposition of the thin films. GN acknowledges support by the Swiss National Science Foundation (SNSF). MI acknowledges support by an Undergraduate Research Opportunity Program (UROP) grant from the University of Colorado. 


\section{REFERENCES}

1. H. Raether, Surface Plasmons on Smooth and Rough Surfaces and on Gratings, (Springer, 1988).

2. W. L. Barnes, A. Dereux, and T. W. Ebbesen, "Surface plasmon subwavelength optics," Nature 424, 824-830 (2003).

3. S. A. Maier, Plasmonics: Fundamentals and Applications (Springer, 2007).

4. B. Liedberg, C. Nylander, and I. Lunstroem, "Surface plasmon resonance for gas detection and biosensing," Sensor. Actuator. 4, 299-304 (1983).

5. J. Homola, S. S. Yee, and G. Gauglitz, "Surface plasmon resonance sensors: review," Sensor. Actuat. B-Chem. 54, 3-15 (1999).

6. M. Li, S. K. Cushing, and N. Wu, "Plasmon-enhanced optical sensors: a review," Analyst 140, 386-406 (2015).

7. R. A. Innes and J. R. Sambles, "Optical characterisation of gold using surface plasmon-polaritons," J. Phys. F: Met. Phys. 17, 277 (1987).

8. J. M. Brockman, B. P. Nelson, and a. R. M. Corn, "Surface Plasmon Resonance Imaging Measurements of Ultrathin Organic Films," Annu. Rev. Phys. Chem. 51, 41-63 (2000).

9. A. G. Notcovich, V. Zhuk, and S. G. Lipson, "Surface plasmon resonance phase imaging," Appl. Phys. Lett. 76, 1665-1667 (2000).

10. B. Vohnsen and D. Valente, "Surface-plasmon-based wavefront sensing," Optica, OPTICA 2, 1024-1027 (2015).

11. R. B. Pettit, J. Silcox, and R. Vincent, "Measurement of surface-plasmon dispersion in oxidized aluminum films," Phys. Rev. B 11, 3116-3123 (1975).

12. H. R. Gwon and S. H. Lee, "Spectral and Angular Responses of Surface Plasmon Resonance Based on the Kretschmann Prism Configuration," Mater. Trans. 51, 1150-1155 (2010).

13. S. C. Kitson, "Full Photonic Band Gap for Surface Modes in the Visible," Phys. Rev. Lett. 77, 2670-2673 (1996).

14. J. D. Swalen, J. G. G. Ii, M. R. Philpott, A. Brillante, I. Pockrand, and R. Santo, "Plasmon surface polariton dispersion by direct optical observation," Am. J. Phys. 48, 669-672 (1980).

15. S. C. Kitson, W. L. Barnes, G. W. Bradberry, and J. R. Sambles, "Surface profile dependence of surface plasmon band gaps on metallic gratings," J. Appl. Phys. 79, 7383-7385 (1996).

16. C. Park, J. Choi, and Y.-H. Cho, "Real-time surface plasmon resonance dispersion imaging with a wide range of incident angles and detection wavelengths," Appl. Opt. 49, 2470-2474 (2010).

17. R. L. Olmon, B. Slovick, T. W. Johnson, D. Shelton, S.-H. Oh, G. D. Boreman, and M. B. Raschke, "Optical dielectric function of gold," Phys. Rev. B 86, 235147 (2012).

18. A. D. Rakic, A. B. Djurisic, J. M. Elazar, and M. L. Majewski, "Optical properties of metallic films for vertical-cavity optoelectronic devices," Appl. Opt. 37, 5271-5283 (1998).

19. www.refractiveindex.info.

20. M. Born and E. Wolf, Principles of Optics: Electromagnetic Theory of Propagation, Interference and Diffraction of Light (Cambridge University Press, 1999).

21. H. J. Simon, D. E. Mitchell, and J. G. Watson, "Surface plasmons in silver films-a novel undergraduate experiment," Am. J. Phys. 43, 630-636 (1975). 